The effect of gender on eye colour variation in European populations and an evaluation of the IrisPlex prediction model

Pietroni, Carlotta; Andersen, Jeppe D; Johansen, Peter; Andersen, Mikkel M; Harder, Stine;

Paulsen, Rasmus; Børsting, Claus; Morling, Niels

Published in:

Forensic science international. Genetics

DOI:

10.1016/j.fsigen.2014.02.002

Publication date:

2014

Citation for published version (APA):

Pietroni, C., Andersen, J. D., Johansen, P., Andersen, M. M., Harder, S., Paulsen, R., Børsting, C., \& Morling, N.

(2014). The effect of gender on eye colour variation in European populations and an evaluation of the IrisPlex prediction model. Forensic science international. Genetics, 11, 1-6. https://doi.org/10.1016/j.fsigen.2014.02.002 


\title{
The effect of gender on eye colour variation in European populations and an evaluation of the IrisPlex prediction model
}

\author{
Carlotta Pietroni $^{\mathrm{a}, 1}$, Jeppe D. Andersen ${ }^{\mathrm{a}, 1, *}$, Peter Johansen ${ }^{\mathrm{a}}$, Mikkel M. Andersen ${ }^{\mathrm{b}}$, \\ Stine Harder ${ }^{c}$, Rasmus Paulsen ${ }^{c}$, Claus Børsting ${ }^{\mathrm{a}}$, Niels Morling ${ }^{\mathrm{a}}$ \\ ${ }^{a}$ Section of Forensic Genetics, Department of Forensic Medicine, Faculty of Health and Medical Sciences, University of Copenhagen, DK-2100 Copenhagen, \\ Denmark \\ ${ }^{\mathrm{b}}$ Department of Mathematical Sciences, Aalborg University, DK-9000 Aalborg, Denmark \\ ${ }^{\mathrm{c}}$ DTU Informatics - Informatics and Mathematical Modeling, Technical University of Denmark, DK-2800 Lyngby, Denmark
}

A R T I C L E I N F O

Article history:

Received 26 November 2013

Received in revised form 29 January 2014

Accepted 2 February 2014

\section{Keywords:}

Forensic genetics

Forensic DNA phenotype

Pigmentation

Eye colour

Gender

\begin{abstract}
A B S T R A C T
In two recent studies of Spanish individuals [1,2], gender was suggested as a factor that contributes to human eye colour variation. However, gender did not improve the predictive accuracy on blue, intermediate and brown eye colours when gender was included in the IrisPlex model [3].

In this study, we investigate the role of gender as a factor that contributes to eye colour variation and suggest that the gender effect on eye colour is population specific.

A total of 230 Italian individuals were typed for the six IrisPlex SNPs (rs12913832, rs1800407, rs12896399, rs1393350, rs16891982 and rs12203592). A quantitative eye colour score (Pixel Index of the Eye: PIE-score) was calculated based on digital eye images using the custom made DIAT software. The results were compared with those of Danish and Swedish population samples.

As expected, we found HERC2 rs12913832 as the main predictor of human eye colour independently of ancestry. Furthermore, we found gender to be significantly associated with quantitative eye colour measurements in the Italian population sample. We found that the association was statistically significant only among Italian individuals typed as heterozygote GA for HERC2 rs12913832. Interestingly, we did not observe the same association in the Danish and Swedish population. This indicated that the gender effect on eye colour is population specific. We estimated the effect of gender on quantitative eye colour in the Italian population sample to be $4.9 \%$. Among gender and the IrisPlex SNPs, gender ranked as the second most important predictor of human eye colour variation in Italians after HERC2 rs12913832. We, furthermore, tested the five lower ranked IrisPlex predictors, and evaluated all possible $3^{6}$ (729) genotype combinations of the IrisPlex assay and their corresponding predictive values using the IrisPlex prediction model [4]. The results suggested that maximum three (rs12913832, rs1800407, rs16891982) of the six IrisPlex SNPs are useful in practical forensic genetic casework.
\end{abstract}

(c) 2014 Elsevier Ireland Ltd. All rights reserved.

\section{Introduction}

Investigations of pigmentary genes have gained much interest in forensic science because of the possible use of genetic markers to predict visible, physical traits of possible offenders by DNA typing of biological samples found at crime scenes. Eye colour is currently the best investigated phenotype for forensic genetic applications. SNP multiplexes dedicated to predict eye colour were published [4-6]. One of these, the IrisPlex assay, was validated for forensic genetic testing [7]. The IrisPlex assay consists of six SNPs

\footnotetext{
* Corresponding author. Tel.: +45 35326916 .

E-mail address: jeppe.dyrberg.andersen@sund.ku.dk (J.D. Andersen).

1 These authors contributed equally to this work.
}

(rs12913832, rs1800407, rs12203592, rs1393350, rs12896399 and rs16891982) that predict qualitative eye colour categories (blue, intermediate and brown). The IrisPlex assay predicts blue and brown eye colours with high accuracy but predict the intermediate eye colours with lower accuracy [4]. Two recent studies $[1,2]$ suggested that gender is contributing to human eye colour variation. Males were found to be more likely to have blue eye colour compared to females, whereas females showed higher frequencies of intermediate and brown eye colour compared to those of males. Similar results were observed for already published male and female eye colour frequency data in Icelandic and Dutch populations [8] but not in Australian [9] and Polish populations [10]. Martinez-Cadenas and co-workers [1] suggested that the prediction success of the IrisPlex model and other models used to predict eye colour phenotypes from DNA genotypes may be 
improved by including gender as a factor. As a reply to MartinezCadenas and co-worker [3], the inventors of the IrisPlex assay tested whether the prediction of categorical eye colour (blue, intermediate and brown) was improved when gender was considered [3]. They used two large IrisPlex datasets with a total of over 9000 European individuals [4,11]. The results showed that gender did not improve the prediction of the eye colour categories blue, intermediate and brown and concluded that gender had minimal effect on eye colour.

In this study, we investigated if gender is associated with a quantitative eye colour measurement in an Italian population sample. We hypothesize that the effect of gender on eye colour is population specific. To test this, we compared the results obtained in the Italian population sample with those of two Northern European populations (a Danish and a Swedish population) from an already published study [12].

\section{Materials and methods}

\subsection{Samples and DNA purification}

Blood samples from 230 unrelated Italian individuals were collected at the Department of Forensic Medicine, University of Copenhagen, Denmark and the U.O. di Patologia Clinica, Laboratorio Analisi del Presidio Ospedaliero di Cantú-Mariano Comense, Azienda Opedaliera Ospedale Sant'Anna di Como, Italy. The study was approved by the Danish Ethical Committee (H-3-2012-023), the Ethical Committee of Azienda Ospedaliera Ospedal Sant́Anna di Como (U.0026484.23-11-2012) and the Ethical Committee of the University of Milan-Bicocca (P.U. 0033373/12). All participants gave signed, informed consent. Samples were labelled ITA1-ITA230 and treated as anonymously donated samples.

DNA was purified from $200 \mu \mathrm{L}$ of blood using the DNA Blood Mini Kit (Qiagen) as recommended by the manufacturer. DNA was eluted in $50 \mu \mathrm{L}$ AE Buffer (Qiagen).

\subsection{Digital photographs and quantitative eye colour}

Photographs were taken at a distance of approximately $10 \mathrm{~cm}$ in "Raw" or "jpeg" format with a Canon EOS 5D Mark V with ISO 800 , shutter $1 / 100$ and AV 18 using a Canon EF $100 \mathrm{~mm}$ f/2.8 L IS USM Macro Lens with manual focus. The white balance of "Raw" format photographs was changed to "Flash" using the Picture style editor software (Canon).

The iris region was automatically extracted from the digital photographs using the custom designed software DIAT and a quantitative eye colour score (Pixel Index of the Eye: PIE-score) was calculated for each individual.

The PIE-score was calculated according to the equation below as previously described [12]:

PIE $=\frac{\begin{array}{l}\text { Number of pixels labeled blue }- \\ \text { Number of pixels labeled brown }\end{array}}{\text { Number of pixels labeled blue }+}$.

The PIE-score ranged from -1 to 1 . The value of -1 was observed when only brown pixels were found in the iris region and the value of 1 was observed when only blue pixels were found in the iris region.

\subsection{Qualitative categorization of eye colour}

Eye colours were assigned according to four categories (blue, light intermediate, dark intermediate and brown) by seven untrained individuals, who were placed in front of the same screen at the same distance and asked to assess the colour category of each eye image. Each eye image was assigned an eye colour category by taking the median of the assessed categories.

For a three colour categorization (blue, intermediate and brown), light intermediate and dark intermediate categories were collapsed into one category (intermediate).

\subsection{SNP-typing}

The six IrisPlex SNPs (rs12913832, rs1800407, rs12203592, rs1393350, rs12896399 and rs16891982) were typed as part of multiplex assay with 32 pigmentary SNPs using the iPLEX ${ }^{\mathbb{R}}$ Gold Kit (Sequenom) as previously described [12].

Allele calling of the SNPs were analyzed in the statistical computing software R v.2.11.0 (http://CRAN.R-project.org/doc/ FAQ/R-FAQ.html; ISBN 3-900051-08-9). The Plate Data File with signal to noise ratios (SNR) and peak heights were exported from TYPER 4.0 (Sequenom) and imported into R. The allele balance ( $A B)$ was calculated in $\mathrm{R}$ as $\mathrm{AB}=$ (Height of Allele 1 - Height of Allele 2)/ (Height of Allele $1+$ Height of Allele 2). We defined parameters for correct allele calling; peak height $>1.0$, SNR $>5$ and $|A B|>0.8$ for homozygotes and $|\mathrm{AB}|<0.2$ for heterozygotes. A value of $|\mathrm{AB}|>0.8$ translates to a genotype call where the peak height of one allele was at least 9 times higher than the peak height of the other allele. $|\mathrm{AB}|<0.2$ translates to a heterozygous genotype call where the peak height of one allele was maximally 1.5 times the peak height of the other allele. Modification of ABs were made for rs12913832 A homozygotes $|A B|>0.75$ and for heterozygotes of rs16891982, $0.1<|\mathrm{AB}|<0.3$. All cluster plots were visually inspected. All samples were run in duplicates.

SNP types were compared between spots and duplicate typing using a custom function (PlateCompare) in R.

\subsection{Statistical analyses}

All statistical calculations were performed using R. Bonferroni correction was applied for multiple comparisons. Independent association of gender and SNPs with the PIE-score was carried out using the non-parametric Wilcoxon rank sum test and the Kruskal-Wallis one-way analysis of variance rank test.

The predictive analysis was performed using a multivariate linear regression model. The adjusted $R^{2}$ [13] was used to select informative predictors and to analyze the prediction accuracy of the model.

\section{Results}

\subsection{Association between gender, the IrisPlex SNPS and eye colour}

A total of 230 Italian individuals were typed for the six IrisPlex SNPs. In 95\% (126 females and 92 males) of the 230 eye images, the iris region was successfully extracted and quantitative measurements of the eye colours (the PIE-score) were performed. It was not possible to calculate a PIE-score for 12 individuals due to poor quality of the eye images mainly due to reflections in the iris area. The association of gender and the six IrisPlex SNPs with the PIEscore was investigated using the non-parametric Kruskal-Wallis one-way analysis of variance rank test. After multiple comparison correction (Bonferroni), the significance threshold was $p=0.007$ when $\alpha=0.05$. We observed a significant associations between HERC2 $\operatorname{rs} 12913832\left(p=2.11 \times 10^{-22}\right), \quad$ SLC45A2 rs16891982 $(p=0.001)$, gender $\left(p=1.61 \times 10^{-5}\right)$ and the PIE-score. We compared the results of the Italian population sample with results from an already published study of Swedish and Danish individuals [12] (Table 1). The Danish and the Swedish individuals were typed for the IrisPlex SNPs and the PIE-score was calculated for each individual. We did not observe any significant association between 
Table 1

Associations of gender and the six IrisPlex SNPs with the PIE-score.

\begin{tabular}{|c|c|c|c|c|}
\hline & \multicolumn{4}{|c|}{ p-value (Kruskal-Wallis) } \\
\hline & Danes $(N=351)$ & Italians $(N=218)$ & Swedes $(N=206)$ & All $(N=776)$ \\
\hline Gender & 0.502 & $1.61 \times 10^{-5}$ & 0.443 & 0.018 \\
\hline rs1393350 (TYR) & $9.90 \times 10^{-5}$ & 0.563 & 0.020 & $9.63 \times 10^{-4}$ \\
\hline rs1800407 (OCA2) & $2.02 \times 10^{-4}$ & 0.316 & 0.003 & $2.05 \times 10^{-9}$ \\
\hline rs12203592 (IRF4) & 0.795 & 0.029 & 0.154 & 0.952 \\
\hline rs12896399 (SLC24A4) & 0.007 & 0.973 & 0.007 & $2.62 \times 10^{-9}$ \\
\hline rs12913832 (HERC2) & $4.28 \times 10^{-39}$ & $2.11 \times 10^{-22}$ & $2.04 \times 10^{-17}$ & $9.57 \times 10^{-108}$ \\
\hline rs16891982 (SLC45A2) & 0.003 & 0.001 & $1.29 \times 10^{-4}$ & $4.17 \times 10^{-14}$ \\
\hline
\end{tabular}

Bold numbers: Significant association after multiple Bonferroni correction $(p<0.007)$.

gender and the PIE-score in the Danes (168 females and 183 males) $(p=0.502)$ nor in the Swedes (134 females and 72 males) $(p=0.443)$.

We observed that HERC2 rs12913832 showed the strongest association with the PIE-score in all three populations. This was in concordance with previous studies in which HERC2 rs12913832 was described to be the main predictor of qualitative [14-16] and quantitative eye colour [17]. To assess possible confounding effects of HERC2 rs12913832 on the gender effect, association tests of gender with the PIE-score were carried out using the nonparametric Wilcoxon-rank-sum test based on the SNP-types of HERC2 rs12913832 (Table 2 and Fig. 1). After Bonferroni correction for multiple comparisons, the significance threshold was $p=0.008$ when $\alpha=0.05$. Gender remained significantly associated with the PIE-score $\left(p=1.05 \times 10^{-5}\right)$ in the Italian individuals that were typed to be heterozygote GA for rs12913832 (Table 2). However, gender was not significantly associated with the PIE-score in individuals typed as either rs $12913832 \mathrm{G}$ or rs12913832 A. None of the Danish or Swedish sample groups showed significant association with gender or the PIE-score. Among Italian rs12913832 GA individuals, females had more brown eye colour (median PIEscore $=-0.93$ ) compared to the eye colour of males (median PIEscore $=-0.56$ ). Among the Italians typed as rs12913832 G, females also had a tendency to have darker eye colours than males. However, the result was not significant $(p=0.045)$ when the Bonferroni corrected threshold of $p=0.008$ was applied.

\subsection{The effect of gender on eye colour in the Italian population sample}

To investigate the effect of gender on the PIE-score in the Italian population sample and hence the eye colour, we performed a multivariate linear regression analysis. We analyzed the predictive values of the six IrisPlex SNPs and gender by using the adjusted $\mathrm{R}^{2}$ to select predictors for the final model. The final model included the SNPs rs12913832, rs1800407, rs16891982, rs12203592 and gender as predictors, whereas rs1393350 and rs12896399 did not show any effect on eye colour. The effect of each of the predictors was evaluated using the $\Delta R^{2}$ (adjusted $R^{2}$ of the model including the specific predictor minus the adjusted $R^{2}$ of the model without the specific predictor but with the other predictors) (Table 3 ). We

Table 2

Association of gender with the PIE-score based on rs12913832.

\begin{tabular}{lllll}
\hline & \multicolumn{4}{l}{$p$-value (Wilcoxon-rank-sum) } \\
\cline { 2 - 5 } & Danes & Italians & Swedes & All three \\
\hline rs12913832 (G) & 0.297 & 0.045 & 0.781 & 0.990 \\
rs12913832 (GA) & 0.381 & $\mathbf{1 . 0 5} \times \mathbf{1 0}^{-\mathbf{5}}$ & 0.721 & $\mathbf{0 . 0 0 2}$ \\
rs12913832 (A) & 0.317 & 0.154 & 0.134 & 0.130
\end{tabular}

Bold numbers: Significant association after multiple Bonferroni correction $(p<0.008)$ found the effect of gender to be $4.9 \%\left(\Delta R^{2}=4.9 \%\right)$ in the Italian population.

\section{Discussion}

\subsection{Gender and eye colour in the Italian population sample}

Gender was previously described to be a significant predictor of quantitative eye colour when eye colour was quantified into the hue and the saturation component of the HSV colour space [17]. However, gender was ranked only no. 14 out of 14 investigated predictors and it was reported to explain only $0.04 \%$ of the hue component variation and $0.09 \%$ of the saturation component variation of the iris. Similarly, we used the PIE-score as a quantitative measurement for eye colour that essentially is based on the saturation component of the HSV colour space [12]. The PIEscore is calculated as the ratio between pixels categorized as blue and pixels categorized as brown in the iris region of a digital eye image. The categorization is based on the saturation component. We previously showed that there is a very high correlation between the PIE-score and human evaluation of the eye colour in the Danish and Swedish sample sets (Spearman correlation $=-0.81)[12]$. We found a high correlation in the Italian sample set (Spearman correlation $=-0.85$ ) (Supplementary Figure 1). We found the effect of gender to be $4.9 \%\left(\Delta R^{2}=4.9 \%\right)$ in the Italian population sample. This was larger than the effect of $0.09 \%\left(\Delta R^{2}=0.09 \%\right)$ presented in a previous study [17]. The most likely explanation for this difference is that there are large gender effects on eye colours between different populations. Our effect was predicted using an Italian population collected in Northern Italy, whereas the previous effect of $0.09 \%$ was based on individuals of Northern European descent (Dutch, British and Australian individuals).

We are aware of the fact that the number of individuals studied was relatively small and limited to only one population. However, the results presented here and by Martinez-Cadenas and coworkers [1,2] support a population specific gender effect on eye colour. To fully explore possible population specific effects, more populations need to be studied. Especially, Mediterranean populations will be interesting.

\subsection{Accuracy of the IrisPlex prediction model in the Italian population sample}

It is not the general assumption that women have darker eye colours than men. Thus, the results presented here and by Martinez-Cadenas and co-workers [1,2] were unexpected. It raises the question whether the gender effect is, although statistically significant in Spanish and Italian population sample sets, relevant for forensic genetic case work in specific populations. Gender was ranked 2nd among the predictors in the Spanish and Italian population samples. On the other hand, the effect may be so small 

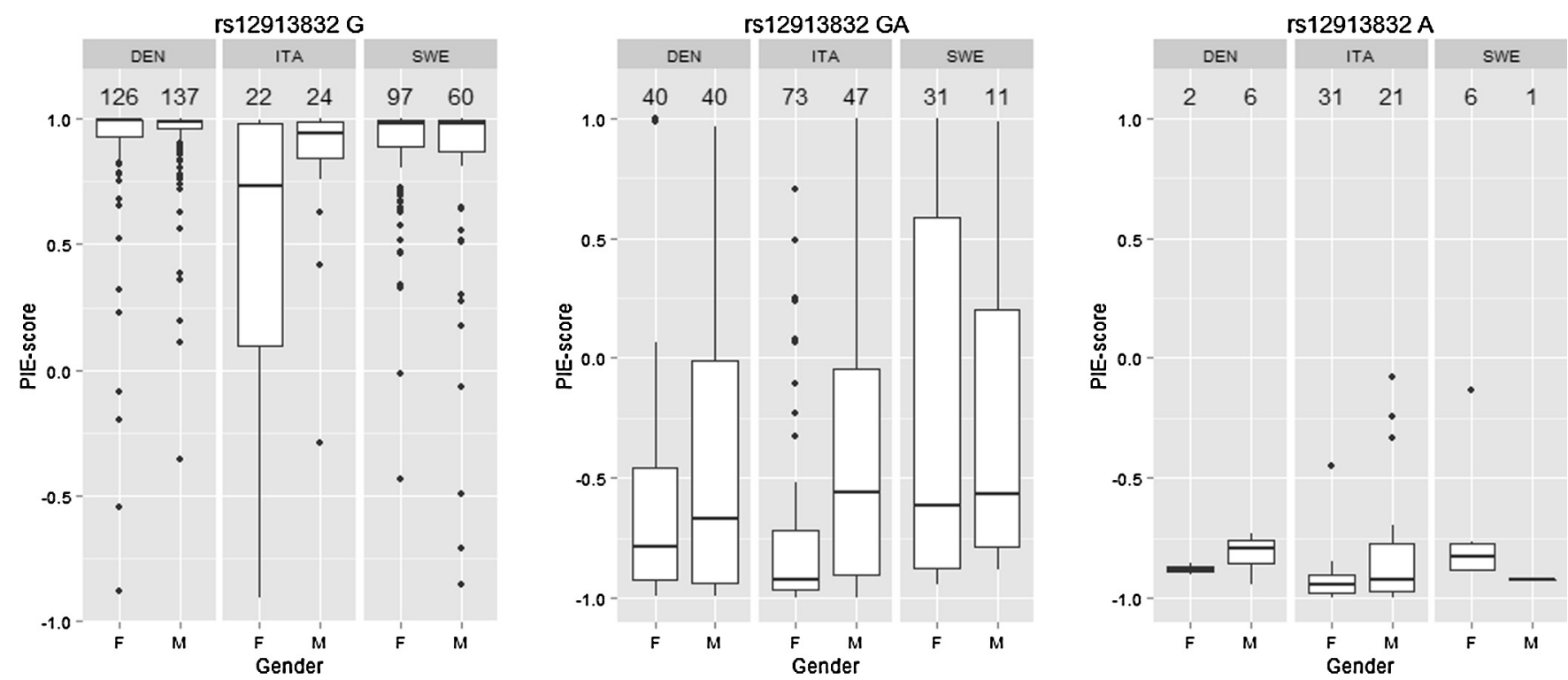

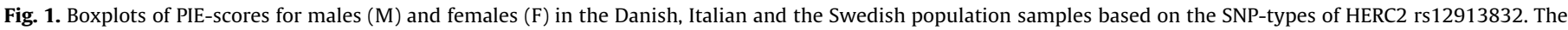
numbers above each boxplot indicate the number of observations.

that it is not relevant for the human perception of eye colour. If so, it may also raise the question whether lower ranked predictors including five of the six IrisPlex SNPs are relevant for the prediction of human perception of eye colour.

To evaluate this, we first tested the accuracy of the IrisPlex prediction model in the Italian population sample set. We used the suggested threshold probability of $p>0.7$ for a conclusive prediction of blue, intermediate or brown eye colour [11] and compared it to a simple prediction model with only HERC2 rs12913832. The predictions of the simple model were calculated using the Snipper classifier [6] that allows prediction of the eye colour based on a subset of the IrisPlex SNPs (in this case, only HERC2 rs12913832). The model predicted blue eye colour for rs12913832 GG individuals and brown eye colour for rs12913832 AA individuals whereas the eye colour of rs12913832 GA individuals was inconclusive (Table 4).

Table 4 shows that the simple prediction model only based on rs12913832 performed as well as the IrisPlex prediction model using all six IrisPlex SNPs in the Italian population sample. This

Table 3

Predictors for quantitative eye colours in the Italian population sample.

\begin{tabular}{llc}
\hline & $p$-value (ANOVA) & $\Delta R^{2} \%$ \\
\hline rs12913832 (HERC2) & $2.2 \times 10^{-16}$ & 51.4 \\
Gender & $1.33 \times 10^{-7}$ & 4.9 \\
rs1800407 (OCA2) & 0.211 & 0.6 \\
rs12203592 (IRF4) & 0.012 & 1 \\
rs16891982 (SLC45A2) & 0.014 & 1.3
\end{tabular}

Table 4

Accuracy of prediction models in the Italian population sample.

\begin{tabular}{lllll}
\hline & & Blue & Intermediate & Brown \\
\hline IrisPlex prediction model $^{\mathrm{a}}$ & Sensitivity & 0.96 & 0 & 1 \\
& Specificity & 0.81 & 1 & 0.55 \\
\multirow{2}{*}{ Snipper (rs12913832) } & & & & 1 \\
& Sensitivity & 1 & - & 0.70 \\
\hline
\end{tabular}

a A total of 143 samples had a prediction probability above 0.7 and 75 samples ( 2 blue, 45 intermediate and 28 brown) were inconclusive $(p<0.7)$.

b A total of 98 samples fulfilled the prediction criteria (LR $>3$ ) and 120 samples (2 blue, 61 intermediate and 57 brown) were inconclusive $(\mathrm{LR}<3)$. indicated that the effect of the lower ranked predictors is too small to influence the predictive accuracy of blue, intermediate and brown eye colours in the Italian population sample.

A similar conclusion was reached for gender when the influence of gender on the predictive capability of the IrisPlex model was analyzed in two large datasets, one dataset including 5348 Dutch Europeans and one dataset including 3840 mixed Europeans [3].

\subsection{The predictive properties of the IrisPlex prediction model}

To further test the predictive properties of the five lower ranked IrisPlex predictors, we evaluated all possible genotype combinations of the IrisPlex assay and their corresponding predictive values using the IrisPlex prediction model [4]. A total of $3^{6}(729)$ different genotype combinations are possible with the six IrisPlex SNPs. Of these, $292(292 / 729,40.1 \%)$ were predicted as blue, 60 $(60 / 729,8.2 \%)$ as intermediate and 377 (377/729, 51.7\%) as brown (Supplementary Table 1 ). If the minimum threshold of $p>0.7$ for a conclusive prediction was applied, all but two genotypes predicted as blue included rs 12913832 GG (Table 5) and only 28 of 243 possible genotypes with rs12913832 GG were inconclusive. All except one of the inconclusive genotype combinations with rs12913832 GG included the rs16891892 CC genotype. rs16891982 is considered to be a major ancestry informative marker (AIM) and the C allele is found in high frequency in African individuals [18]. A functional role of rs16891982 in eye colour and pigmentation has not been revealed. This is contrary to rs12913832, where the allele rs12913832 G was shown to reduce the transcription of OCA2 and hence result in a lower pigmentation level [19]. It is questionable whether rs16891982 CC can alter the eye colour phenotype determined by rs12913832 GG. Furthermore, it is highly unlikely to encounter a person with the genotype

Table 5

Prediction outcomes of the IrisPlex prediction model.

\begin{tabular}{lccc}
\hline & rs12913832 GG & rs12913832 GA & rs12913832 AA \\
\hline Blue & $215(242)$ & $2(50)$ & $0(0)$ \\
Intermediate & $0(0)$ & $0(54)$ & $0(6)$ \\
Brown & $0(1)$ & $59(139)$ & $212(237)$ \\
Inconclusive $(p<0.7)$ & 28 & 182 & 31 \\
\hline
\end{tabular}

Number in parentheses: Prediction without a threshold of $p>0.7$. 


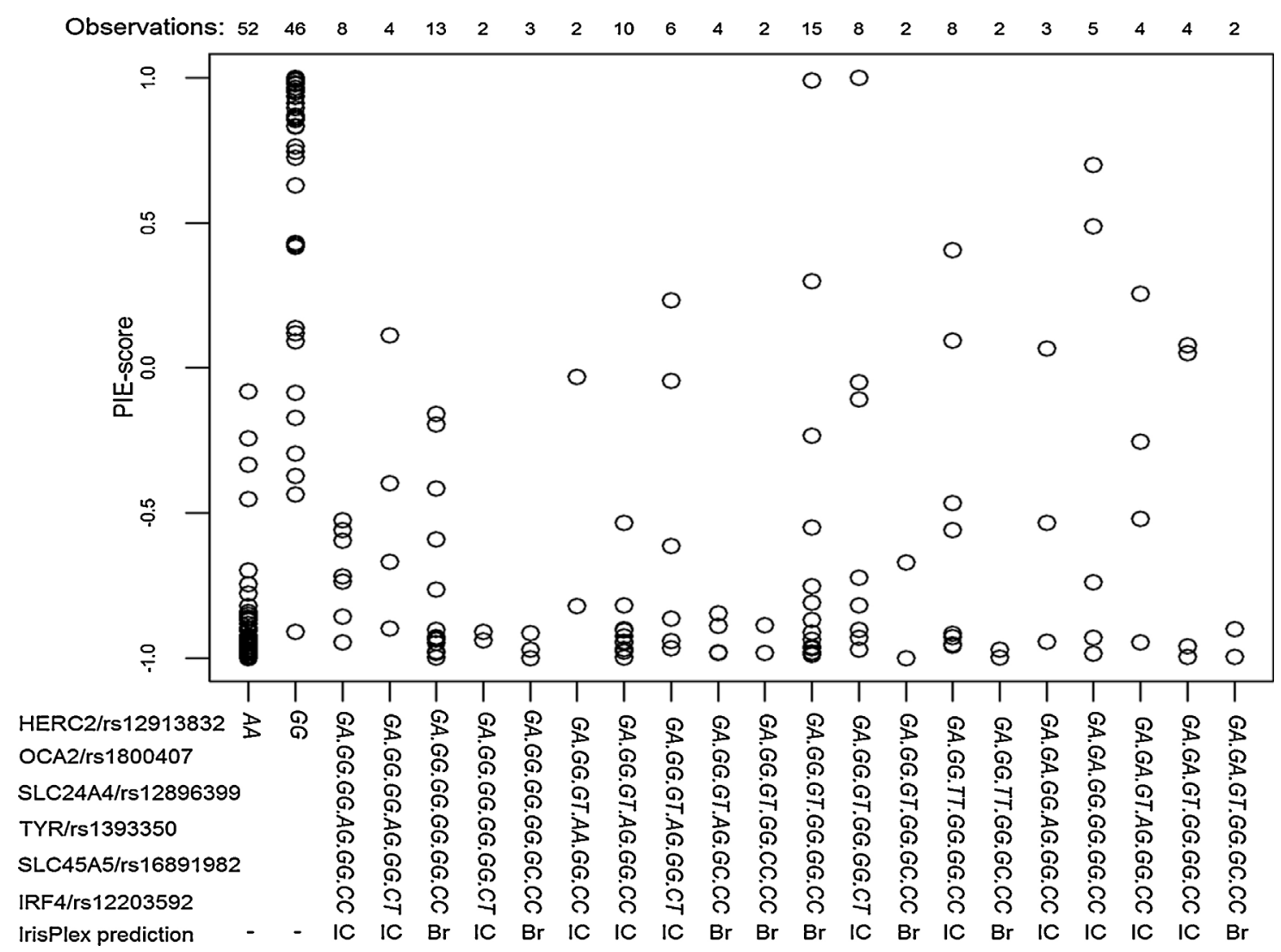

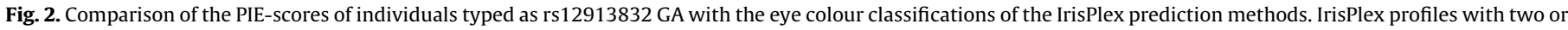

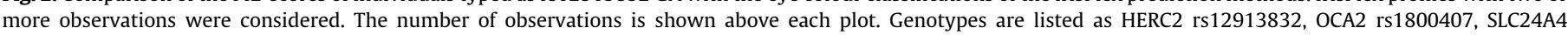

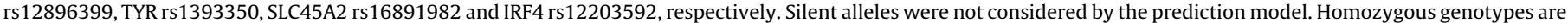

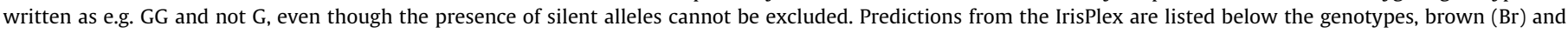
inconclusive (IC).

rs128913832 GG and rs16891982 CC because the rs128913832 G allele is almost exclusively found in Europeans whereas the rs $16891982 \mathrm{C}$ is typically found in Africans. Thus, for all practically purposes, the rs12913832 GG genotype predicts blue eye colours irrespective of the lower ranged predictors in the IrisPlex.

Among the 243 genotype combinations with rs12913832 AA, 212 reached the threshold of $p>0.7$ and were predicted as brown, whereas 31 genotypes were inconclusive (Table 5). Interestingly, rs 1800407 AA was included in 27 of the 31 inconclusive genotype combinations with rs12913832 AA, and rs1800407 GA was included in the remaining four combinations. rs1800407 is a missense mutation in OCA2 located $135 \mathrm{kp}$ downstream from rs 12913832 . The haplotypes of this region were previously suggested to increase eye colour prediction $[5,6,20]$. It was suggested $[12,21]$ that the OCA2 allele rs $1800407 \mathrm{~A}$ decreased the pigmentation level in the eye when found in cis phase with the HERC2 allele rs12913832 A, whereas the OCA2 allele rs $1800407 \mathrm{~A}$ found in cis phase with the HERC2 allele rs12913832 G will display little effect on the eye colour because the transcription of OCA2 is reduced.

It is well known that the eye colours of individuals typed as rs12913832 GA are the most difficult to predict [4]. The Italian individuals with rs12913832 GA had large variations in PIE-scores (Fig. 2). Also, Danish and Swedish individuals with rs12913832 GA had eye colours ranging from blue to brown [12]. If the threshold of $p>0.7$ was applied to the IrisPlex model, 182 of the 243 genotypes were inconclusive, 59 genotypes were predicted as brown and only two as blue. The two genotype combinations predicted as blue both included rs1800407 AA ( $p=0.71$ for both). Of the 59 combinations predicted as brown, 41 combinations included rs16891982 CC and 16 combinations included rs16891982 CG.
The average prediction probability of the 61 genotypes with $p>0.7$ was only 0.82 . Only 10 genotypes, all including rs 16891982 CC, had $p>0.9$. Thus, prediction of eye colour in the rs12913832 GA group is weak except for individuals of presumably African origin. Supplementary Table 1 lists all the IrisPlex genotype combinations, the European population frequency and the prediction probabilities for blue, intermediate and brown eye colour.

In conclusion, the analysis of the IrisPlex prediction model indicated that three of the six SNPs are important for prediction of eye colour. Of these, only rs12913832 has a known biological function in pigmentation [19]. Taking this and the similar performance of the IrisPlex prediction model and the prediction using only rs12913832 into consideration (Table 4), and following the tradition of conservative statistical weight calculations in the field of forensic genetics, it seems reasonable to restrict the prediction of eye colour to just one SNP, rs12913832. rs12913832 GG predicts blue eye colour, rs12913832 AA predicts brown eye colour and the prediction of the eye colours of individuals typed as rs12913832 GA is inconclusive. Future investigation may show whether rs 1800407 should be considered since it potentially can alter the functionality of OCA2 and hence the eye pigmentation. Also, it is still to be shown if rs16891982 is an AIM for African individuals or has a functional role in eye colour regulation.

\section{Role of funding}

Carlotta Pietroni was supported by the ERASMUS programme (N. 2012-1-IT2-ERA02-38290) and the University of Milan-Bicocca (Italy). 


\section{Acknowledgements}

Dr. Gianni Giana, Dr. Enrico Minotti, Dr. Silvia Sarpau and all of the staff of the O.U. di Patologia Clinica, Lab. Analisi di CantúMariano Comense, Azienda Ospedaliera Sant'Anna di Como (Italy) for their technical support in the collection of samples.

\section{Appendix A. Supplementary data}

Supplementary data associated with this article can be found, in the online version, at doi:10.1016/j.fsigen.2014.02.002.

\section{References}

[1] C. Martinez-Cadenas, M. Pena-Chilet, M. Ibarrola-Villava, G. Ribas, Gender is a major factor explaining discrepancies in eye colour prediction based on HERC2/OCA2 genotype and the IrisPlex model, Forensic Sci. Int. Genet. 7 (4) (2013).

[2] C. Martinez-Cadenas, M. Pena-Chilet, M.J. Llorca-Cardeno, R. Cervera, M. IbarrolaVillava, G. Ribas, Gender and eye colour prediction discrepancies: a reply to criticisms, Forensic Sci. Int. Genet. (2013), http://dx.doi.org/10.1016/j.fsigen.2013.10.002.

[3] F. Liu, S. Walsh, M. Kayser, Of sex and IrisPlex eye colour prediction: a reply to Martinez-Cadenas et al., Forensic Sci. Int. Genet. (2013), http://dx.doi.org/ 10.1016/j.fsigen.2013.06.006.

[4] S. Walsh, F. Liu, K.N. Ballantyne, M. van Oven, O. Lao, M. Kayser, IrisPlex: a sensitive DNA tool for accurate prediction of blue and brown eye colour in the absence of ancestry information, Forensic Sci. Int. 3 (2010) 170-180.

[5] J. Mengel-From, C. Borsting, J.J. Sanchez, H. Eiberg, N. Morling, Human eye colour and HERC2, OCA2 and MATP, Forensic Sci. Int. Genet. 4 (2010) 323-328.

[6] Y. Ruiz, C. Phillips, A. Gomez-Tato, J. Alvarez-Dios, C.M. Casares de, R. Cruz, O. Maronas, J. Sochtig, M. Fondevila, M.J. Rodriguez-Cid, A. Carracedo, M.V. Lareu, Further development of forensic eye color predictive tests, Forensic Sci. Int. Genet. 7 (1) (2013) 28-40.

[7] S. Walsh, A. Lindenbergh, S.B. Zuniga, T. Sijen, K.P. de, M. Kayser, K.N. Ballantyne, Developmental validation of the IrisPlex system: determination of blue and brown iris colour for forensic intelligence, Forensic Sci. Int. Genet. 5 (5) (2011) 464-471.

[8] P. Sulem, D.F. Gudbjartsson, S.N. Stacey, A. Helgason, T. Rafnar, K.P. Magnusson, A. Manolescu, A. Karason, A. Palsson, G. Thorleifsson, M. Jakobsdottir, S. Steinberg, S. Palsson, F. Jonasson, B. Sigurgeirsson, K. Thorisdottir, R. Ragnarsson, K.R. Benediktsdottir, K.K. Aben, L.A. Kiemeney, J.H. Olafsson, J. Gulcher, A. Kong,
U. Thorsteinsdottir, K. Stefansson, Genetic determinants of hair, eye and skin pigmentation in Europeans, Nat.Genet. 39 (12) (2007) 1443-1452.

[9] D.L. Duffy, G.W. Montgomery, W. Chen, Z.Z. Zhao, L. Le, M.R. James, N.K. Hayward, N.G. Martin, R.A. Sturm, A three-single-nucleotide polymorphism haplotype in intron 1 of OCA2 explains most human eye-color variation, Am. J. Hum. Genet. 80 (2) (2007) 241-252.

[10] W. Branicki, U. Brudnik, A. Wojas-Pelc, Interactions between HERC2, OCA2 and MC1R may influence human pigmentation phenotype, Ann. Hum. Genet. 73 (2) (2009) 160-170

[11] S. Walsh, A. Wollstein, F. Liu, U. Chakravarthy, M. Rahu, J.H. Seland, G. Soubrane, L. Tomazzoli, F. Topouzis, J.R. Vingerling, J. Vioque, A.E. Fletcher, K.N. Ballantyne, M Kayser, DNA-based eye colour prediction across Europe with the IrisPlex system, Forensic Sci. Int. Genet. 6 (3) (2011) 330-340.

[12] J.D. Andersen, P. Johansen, S. Harder, S.R. Christoffersen, M. Delgado, S.T. Henriksen, M.M. Nielsen, E. Sørensen, H. Ullum, T. Hansen, A.L. Dahl, R.R. Paulsen, C. Borsting, N. Morling, Genetic analyses of the human eye colour using a novel objective method for eye colour classification, Forensic Sci. Int. Genet. 7 (5) (2013) 508-515.

[13] P. Yin, X. Fan, Estimating R2 shrinkage in multiple regression: a comparison of different analytical methods, J. Exp. Educ. 69 (2) (2001) 203-244.

[14] H. Eiberg, J. Troelsen, M. Nielsen, A. Mikkelsen, J. Mengel-From, K.W. Kjaer, L. Hansen, Blue eye color in humans may be caused by a perfectly associated founder mutation in a regulatory element located within the HERC2 gene inhibiting OCA2 expression, Hum. Genet. 123 (2008) 177-187.

[15] R.A. Sturm, D.L. Duffy, Z.Z. Zhao, F.P. Leite, M.S. Stark, N.K. Hayward, N.G. Martin, G.W. Montgomery, A single SNP in an evolutionary conserved region within intron 86 of the HERC2 gene determines human blue-brown eye color, Am. J. Hum. Genet. 82 (2) (2008) 424-431.

[16] F. Liu, D.K. van, J.R. Vingerling, A. Hofman, A.G. Uitterlinden, A.C. Janssens, M. Kayser, Eye color and the prediction of complex phenotypes from genotypes, Curr. Biol. 19 (5) (2009) R192-R193.

[17] F. Liu, A. Wollstein, P.G. Hysi, G.A. Ankra-Badu, T.D. Spector, D. Park, G. Zhu, M. Larsson, D.L. Duffy, G.W. Montgomery, D.A. Mackey, S. Walsh, O. Lao, A. Hofman, F. Rivadeneira, J.R. Vingerling, A.G. Uitterlinden, N.G. Martin, C.J. Hammond, M. Kayser, Digital quantification of human eye color highlights genetic association of three new loci, PLoS. Genet. 6 (2010) e1000934-e1000940.

[18] C. Phillips, L. Prieto, M. Fondevila, A Salas, A Gomez-Tato, J. Alvarez-Dios, A Alonso, A. Blanco-Verea, M. Brion, M. Montesino, A. Carracedo, M.V. Lareu, Ancestry analysis in the 11-M Madrid bomb attack investigation, PLoS One 4 (8) (2009) e6583-e6590.

[19] M. Visser, M. Kayser, R.J. Palstra, HERC2 rs12913832 modulates human pigmentation by attenuating chromatin-loop formation between a long-range enhancer and the OCA2 promoter, Genome Res. 22 (3) (2012) 446-455.

[20] E. Pospiech, J. Draus-Barini, T. Kupiec, A. Wojas-Pelc, W. Branicki, Gene-gene interactions contribute to eye colour variation in humans, J. Hum. Genet. 56 (6) (2011) 447-455.

[21] D. White, M. Rabago-Smith, Genotype-phenotype associations and human eye color, J. Hum. Genet. 56 (1) (2011) 5-7. 